\title{
Rôle de la vision et du contexte visuel dans la construction du sens - The Oval Portrait
}

Séverine Letalleur-Sommer

\section{Q OpenEdition \\ 1 Journals}

Édition électronique

URL : http://journals.openedition.org/esa/1614

DOI : $10.4000 /$ esa. 1614

ISSN : 2650-2623

Éditeur

Société de stylistique anglaise

Édition imprimée

Date de publication : 31 décembre 2011

Pagination : 59-73

ISSN : 2116-1747

\section{Référence électronique}

Séverine Letalleur-Sommer, «Rôle de la vision et du contexte visuel dans la construction du sens - The Oval Portrait », Études de stylistique anglaise [En ligne], 3 | 2011, mis en ligne le 27 novembre 2018, consulté le 20 avril 2019. URL : http://journals.openedition.org/esa/1614 ; DOI : 10.4000/esa.1614 


\title{
ROLE DE LA VISION ET DU CONTEXTE VISUEL DANS LA CONSTRUCTION DU SENS - THE OVAL PORTRAIT
}

\author{
Séverine Letalleur-Sommer \\ Université de Paris Ouest-Nanterre La Défense - CREA - EA 370
}

\begin{abstract}
The notion of "context" can be understood as what lies outside of the text proper; notably, what the text signifies and generates in terms of mental images. Here, my aim is to examine the text's visual dimension, in particular the way the reader's gaze adjusts words to the images they trigger. In order to do so, I focus on Poe's famous short story "The Oval Portrait". Because of its specific structure (an embedded story in the third person singular added to the initial narrative in the first person) and the theme it tackles (the story of a painting that absorbs life from its model), the fantastic tale can be interpreted as revelatory of the semiotic cogency of words and images. Beyond, it illustrates how a text, with apparently more limited means, is capable of the same immediate effects as pictorial signs.
\end{abstract}

Keywords: semiotics, pictorial signs, two-dimensional, linearity, indexicality, iconicity, logogen, imagen, metadiscourse, text/image

Récit fantastique s'articulant autour de l'évocation d'une œuvre picturale «plus vraie que nature », la nouvelle de Poe «The Oval portrait» sous-tend une réflexion sur les liens qui unissent texte et contexte, le signe ${ }^{2}$ et son

1 Cet article, accepté par le comité de lecture, est issu de la communication présentée en mai 2011 dans l'atelier de stylistique lors du $51^{\text {ème }}$ Congrès de la SAES à Paris Diderot et Paris Sorbonne Nouvelle.

2 J'utilise ici la terminologie guillaumienne et non saussurienne, Guillaume procédant en effet à une correction en faisant remarquer que la relation signifiant/signifié est erronée car c'est la relation signe/signifié qui est signifiante, porteuse de sens ; j'emploie donc ici le terme «signe » dans ce qui dans la terminologie saussurienne est compris sous le terme de «signifiant ». 
signifié, le concret et l'abstrait. Le contexte renvoie à tout ce qui n'est pas le texte mais qui forcément l'accompagne, tout ce qui s'y lit et le relie à autre chose: un autre texte par exemple comme c'est le cas ici, ou une forme intrinsèquement autre à l'instar de ce portrait dont le narrateur cherche à résoudre l'énigme. De fait, si le conte atteint son apogée au moment où le narrateur est sidéré par le visage qu'il découvre dans la pénombre d'une alcôve, il contient aussi un fragment de texte enchâssé, signalé entre guillemets, que l'on est amené à lire comme le narrateur le découvre. Ce deuxième récit se veut une explicitation a posteriori du premier moment de stupeur mutique; en ce sens, il en incarne à la fois le contexte et le co-texte. Il est aussi contenu par lui puisque sa découverte constitue un élément de l'intrigue. Cette imbrication se traduit donc à la fois du point de vue de la diégèse, de celui de son occupation d'un espace distinct sur la page mais aussi du point de vue chronologique, le moment ultérieur (ici situé au début du conte) recélant d'une certaine façon les événements dont il est issu.

L'économie textuelle atypique et le thème abordé, l'ambition folle d'un peintre s'obstinant à vouloir injecter la vie dans ce dont elle est par définition absente - le signe qui la représente -, permet d'envisager le conte comme une réponse poétique aux questions de construction du sens visuel et textuel que se pose le sémioticien. Outre une réflexion sur la création artistique et le statut de l'art, il interroge le mode de fonctionnement des signes en général. Or, je souhaiterais montrer que les choix stylistiques génèrent précisément des effets esthétiques à portée métadiscursive. Tout en caractérisant la forme littéraire, notamment dans ce qui la distingue de l'image et dans ce qui l'en rapproche, ils agissent comme des révélateurs de la façon dont le sens se construit, de la perception à sa conceptualisation en passant par sa représentation. Dès lors, ce qui relève de la périphérie et du contexte de l'œuvre littéraire, les rouages qui la régissent, le pictural, les images générées, les réflexions sur la forme, se voient ici recentrés au cœur du récit alors même que l'intrigue ne serait finalement qu'un prétexte, un leurre narratif ; un peu comme ce portrait dont le titre ne nous annonce que la forme du cadre et non ce qui s'y loge.

\section{Portrait du texte}

Le conte de Poe se veut dès le départ affaire de style et d'esthétique, un travail raffiné autour de mots, de phonèmes et de figures aussi élaborées que les arabesques qui encadrent les nombreuses peintures évoquées dans le récit. Car, à la question centrale que se pose le premier narrateur, celle de l'effet de l'art, « the true secret of its effect » (Poe 1842, 204), capable de susciter l'émotion la plus vive par le truchement d'une forme figée («[...] which had so suddenly and so vehemently moved me» [189]), Poe réplique par un néologisme, une 
forme verbale innovante signalée en italiques dans le texte : «lifelikeliness » (189). Le jeu allitératif, assonantique et graphique célèbre là une langue fertile, capable de prolifération et de réverbération à partir de quelques phonèmes et/ou graphèmes, contrairement au portrait, étroitement circonscrit dans son cadre. Il ne s'agit pas ici d'une reprise banale de la réalité, mais de la variation et de la répétition du même dans l'altérité : life/like/li, le même et l'autre. Il est à noter que la réalisation vocalique du dernier $i$ constitue une monophtongue contrairement aux deux premiers. L'addition interne de la syllabe -li ajoute à l'effet de redondance phonologique. Le suffixe -ness vient rompre la série de variations en scellant la nominalisation de l'ensemble. La présence de likeliness signale que l'on reste dans un rapport d'approximation de la forme et non de conformité totale. L'épithète "absolute » indique simplement que l'on touche là aux limites du possible : «the absolute lifelikeliness of the expression». L'absolu est relatif. De fait, la thématique picturale bien qu'omniprésente, ne trouve ici d'expression que textuelle donc uniquement dans ce qu'elle n'est pas, ce qui interdit tout isomorphisme. Aussi l'hapax de Poe est-il une façon de suggérer les chemins versatiles qu'emprunte la pensée dans sa quête de sens, de l'image au texte et vice-versa. En jaugeant l'élasticité du langage, Poe en explore les ressources sémiotiques et les limites sémantiques.

Si le premier narrateur dispose tout à la fois de l'image et du texte l'histoire relatée dans un livret explicatif trouvé à proximité - pour se faire une idée du modèle qui l'a inspiré et que plus personne ne peut atteindre, le lecteur, en revanche, ne dispose que du texte pour imaginer à quoi ressemble le portrait, et n'a la lettre pour accéder à l'image. La nouvelle est donc d'abord affaire de texte et par là seulement, un métadiscours sur l'efficace sémiotique des deux modes représentationnels que sont la peinture d'une part, et le conte de l'autre. Et ce, d'autant que ce processus ascendant, de la perception visuelle à sa transposition textuelle, traduit en réalité un mouvement inverse où l'auteur puise dans un ensemble d'images endogènes (images mentales) pour conférer par l'écriture une réalité exogène à ses pensées à la surface du papier. Afin d'explorer ce phénomène plus en détails, il convient d'apporter quelques précisions concernant la nature spécifique du texte et de l'image ; là encore, la nouvelle de Poe contient des phénomènes stylistiques tout à fait éclairants.

\section{Texte et image : entre rivalité, dialogue et inversion des rôles}

Bien qu'ils présentent des affinités, les deux modes d'expression ont longtemps été considérés comme rivaux du fait de leur nature antagonique.

Les signes linguistiques arbitrairement corrélés à leur contenu, fonctionnent sur le mode de la mise à distance de ce qu'ils désignent symboliquement, comme par rejet. Cela m'a d'ailleurs amenée dans un précédent article à étoffer 
la définition de la notion d'indexicalité initialement proposée par Charaudeau et Maingueneau ( «Une expression n'a de sens qu'en référence au contexte d'énonciation » [2002, 236]), dans les termes suivants :

Cette faculté qu'a le discours - l'usage de la langue en contexte - de par son caractère unidimensionnel, d'être pure visée, pure désignation d'autre chose à la fois dans le cotexte et dans le contexte [...] de par son dynamisme, chaque mot de l'énoncé désigne celui qui le suit en même temps qu'il renvoie à son signifié. Pour cette raison, les signes linguistiques présentent une dimension doublement indexicale. (Letalleur-Sommer 2009, 275)

Du fait du double cinétisme nécessaire à leur appréhension, les signes linguistiques sont donc plus contraints séquentiellement que ne le sont les images, considérées comme des phénomènes holistiques et ce, bien que l'on puisse bien entendu explorer l'image en la décomposant ou aborder le texte, de façon plus globale, à partir du reflet bidimensionnel que la page imprimée offre à la rétine, auquel cas l'on peut revenir en arrière, sauter des lignes ou considérer plusieurs signes simultanément :

La liberté dans l'interprétation qu'autorise la non-codification du langage graphique va de pair avec une interprétation à la fois lente, puisque moins prévoyante, et holistique liée à une vision d'ensemble immédiate. (Van de Wiele 2005, 3)

Les signes figuratifs entretiennent par ailleurs un rapport d'iconicité avec leur signifié et fonctionnent donc plus volontiers sur le mode de la substitution que sur celui de l'indexicalité et du transfert - le thème de la vignette, récurrent dans la nouvelle, renvoie d'ailleurs étymologiquement à la feuille de vigne qui, précisément, recouvre et occulte (Lecercle 1990, 84). Déplacement d'une part, remplacement et recouvrement de l'autre. C'est bien là le double phénomène auquel la nouvelle nous confronte avec cette curieuse transposition narrative qui nous fait passer du je homodiégétique du premier récit au $i l$ hétérodiégetique du second, et avec ce processus où le modèle se voit intégralement supplanté par la copie qui lui ôte jusqu'au dernier souffle.

La notion de rivalité entre deux arts à laquelle tend cette altérité foncière des deux formes est explicitement mentionnée au sein du récit: «the Art that was her rival » (Poe 1842, 204). La peinture incarne ici de façon métonymique l'Art en tant qu'idéal de la forme, puisque la notion est graphiquement signalée par une majuscule, mais elle s'incarne aussi métonymiquement tout entière dans le portrait, générant un phénomène d'emboîtement caractéristique de la nouvelle. Or, dans cette lutte acharnée à laquelle se livrent Art et Nature, la beauté naturelle, spontanée d'une jeune fille à peine sortie de l'enfance («a young girl just ripening into womanhood » [Poe 1842, 203]), de ses jeux et de sa gaieté, est littéralement happée par le portrait. Le combat semble perdu d'avance. Il n'en est rien. Poe renverse la chronologie, il commence par décrire 
le funeste reflet («the immortal beauty of its countenance »), le modèle original n'apparaissant qu'ultérieurement et de façon insistante grâce à l'anaphore du syntagme : "a maiden of rarest beauty, and not more lovely than full of glee ». Par ce jeu sur les sons et cette manipulation de la structure narrative, il nous met en garde contre toute simplification hâtive qui consisterait à réduire le langage à son unidimensionnalité et à l'arbitraire des signes qui le composent. Car ici c'est bien la langue qui l'emporte sur le portrait, la langue, souple et vivante à l'image de cette jeune fille emplie d'allégresse, la langue capable de faire retentir un son cristallin là où la peinture n'offre plus qu'un regard prisonnier. Assonance du phonème /i/ et allitération de la voyelle latérale [1] vibrent et créent un contraste avec le segment allitératif composé de consonnes occlusives signalant la rigidité du cadre : «the immortal beauty of its countenance ». La langue, capable d'accomplir un trajet à rebours, d'inverser le cours des choses, se contredit. Elle imite et réinvente le monde en en reproduisant les sons de façon à la fois fidèle et innovante.

Poe ne s'en tient donc pas à une confrontation binaire de la nature des signes. Bien au contraire, il les extrait de leur carcan: le langage imite, la peinture décompose. Ainsi, son écriture révèle le caractère complexe du jeu texte/image au sein duquel chacun revêt les attributs de l'autre pour contribuer différemment et/ou de manière similaire à l'émergence d'une vision d'ensemble puis à l'identification et au prélèvement de fragments. De fait, les entités linguistiques consécutives génèrent des images mentales elles-mêmes parcellaires qu'il faut ensuite réinscrire dans un ensemble cohérent : «the arms, the bosom and even the ends of the hair melted imperceptibly into the vague deep shadow which formed the background of the whole » (Poe 1842, 204). Ici, la structure paratactique rappelle la forme du blason, laquelle implique une présentation métonymique et symbolique du corps féminin. La juxtaposition sans lien logique accentue l'effet de fragmentation du corps. Les constructions en of alternent. S'enchaînent consécution linéaire puis isolement de formes («the ends of the hair») à partir d'un tout dont elles ne se détachent nettement que linguistiquement (of a pour étymologie off) et non visuellement : où commence la pointe des cheveux? Le texte produit alors immanquablement des images endogènes qui, contrairement aux images exogènes de type pictural (image concrète exposée sur un support matériel et donc susceptible d'être partagée par tous), ont pour caractéristique leur plasticité puisqu'elles se transforment et s'enrichissent au fil de la lecture. Ceci n'exclut pas que des synthèses ponctuelles soient indispensables à l'émergence signifiante («the whole»). Simplement, cela se fait de l'intérieur, de façon centripète et en dépit de l'absence de cadre, notamment grâce à la concentration de l'attention initiale sur la lettre ou le mot, concentration rendue d'autant plus impérieuse que le signe est arbitraire : 
But how does the self arise? How do we continually emerge from our sensations [...]? Woolf realized that the self emerges via the act of attention. We bind together our sensory parts by experiencing them from a particular point of view (Lehrer 2008, 181)

De même, la perception globale du tableau donne toujours lieu à une dissection visuelle permettant à des entités de se détacher de l'ensemble. Cela se fait par un phénomène d'accommodation visuelle avec mise en relief d'un élément porté au premier plan, et rejet simultané dans l'arrière-plan de tout le reste («the vague deep shadow which formed the background»), donc par le biais d'un traitement tridimensionnel de l'image bidimensionnelle. On pourrait dire que dans le cas particulier du déchiffrement d'un texte décrivant linéairement le parcours visuel d'une œuvre picturale, se produit un mouvement de protension mais aussi d'étirement vers la droite du fait de la trajectoire qu'impliquent les mécanismes de lecture et d'intégration hiérarchisée des éléments de sens (du phonème ou graphème, au morphème à la phrase et au texte). Ainsi le fait de n'avoir accès à l'image que par la ligne d'écriture suppose que l'on prenne en compte la collusion vectorielle de deux mouvements : avant/arrière - gauche/ droite, le linéaire primant sur le balayage aléatoire. Les phénomènes de retour en arrière n'en sont cependant pas absents mais ils interviennent, comme nous l'avons vu, à une échelle supérieure notamment grâce aux analepses. D'ailleurs, pour Ann Lecercle (1990, 77-91), la fin de la nouvelle résiderait dans le roman d'Ann Radcliffe, The Mysteries of Udolpho, antérieur de quarante-huit années au récit de Poe et explicitement signalé dans le texte (op. cit., 86).

A partir de ces conclusions, nous comprenons que l'intérêt sémiotique de la nouvelle de Poe réside dans le fait que s'y dégage un discours sur le rapport texte/image uniquement pour et par le texte, ce qui signifie naturellement que l'accès à l'image y est symbolique (le texte renvoie ici à ce qui n'est pas lui) mais surtout ne produit que des images endogènes instables et privées. En retour, l'objet-texte, offert au regard de tous, circule dans l'espace social (Belting 2004, 18), tout en se dédoublant par isomorphisme: le passage enchâssé incarnant, concrètement et thématiquement, une reproduction en miniature du premier. On assiste ainsi à l'établissement d'une configuration particulière où le texte comprend une dimension exogène ; il fixe et constitue donc un cadre au sein duquel s'esquisse une image endogène et dynamique, une image vivante.

\section{Lecture incarnée du texte : paradoxe du portrait sans cadre}

Les études récentes portant sur la lecture offrent un éclairage nouveau sur le phénomène décrit ci-dessus. Elles montrent par exemple que le lecteur ne peut à la fois se concentrer sur le signe imprimé et sur son contenu sémantique. L'un prime sur l'autre, et il y a donc dès le départ une oscillation signe/signifié, lettre/image endogène, texte/image : 
In reading, it is somewhat difficult to visually process the print and extensively visualize its semantic content at the same time, particularly for the unskilled reader. Either the reader tends to slow down or the number of oral reading miscues increases. (Sadoski \& Paivio 2004, 6)

On sait par ailleurs que l'apprentissage de la lecture est un processus long et difficile. De fait, l'œil doit d'abord s'adapter à ce type de décryptage linéaire, très distinct de la façon dont on observe une scène ou un paysage par balayage aléatoire quasi-balistique. On observe une visée puis une focalisation, processus qui rappelle un passage de la nouvelle dans lequel le narrateur modifie l'orientation du candélabre afin de mieux distinguer les pages de son livre sans anticiper sur ce qu'il va découvrir («But the action produced an effect altogether unanticipated » [188]). L'œil est contraint par un linéaire où l'ordre dans lequel se succèdent les signes joue un rôle de premier plan (c'est pourquoi l'enfant s'aide en suivant la ligne du doigt), ce qui n'était pas le cas avant :

There is a relatively high number of large saccades, a great deal of recursion, and nothing at all that should make it easy for a subject to keep precise track of the temporal track order in which any search of the visual field has been executed, that is, no mapping of spatial order into temporal sequence. But this is just the task that faces the child when he first learns to read. He has to put together letter into words by a sequence of small adjacent fixations. (Hochberg \& Brooks 2007, 140)

Par l'œil, l'on doit ensuite articuler une parole muette, au sens où l'entend Dehaene qui montre que la coexistence de «deux voies de la lecture » : l'une qui passe par la reconstitution phonologique pour ensuite accéder au sens, l'autre qui passe par le sens afin de rétablir la prononciation du mot $(2007,68)$. Il faut apprendre à se mouvoir virtuellement au fil du linéaire, comme si à chaque lettre correspondait une avancée où le moi se faisait autre en se projetant dans le signe. Je déduis cette projection du fait que la lecture requiert la mobilisation de facultés mentales et motrices à un degré élevé. Il y a une intentionnalité du voir et du savoir (j'ai évoqué plus haut une «concentration impérieuse»), une volonté de créer du sens qui s'exprime dans la lecture comme dans toute activité perceptive. Les phénomènes de subrogation ne sont donc pas exclusivement picturaux.

Dans le texte de Poe, vision et intentionnalité sont étroitement corrélées. Ainsi le verbe frown est utilisé à propos du mélange de grandeur et de mélancolie émanant des nobles bâtisses érigées sur les contreforts des Apennins. La métaphore signale à la fois un changement d'échelle où le point de vue est celui d'un narrateur/spectateur en situation de survol, capable d'embrasser du regard de vastes étendues mais aussi un effort physique de tension visuelle nécessaire à la perception d'éléments situés trop loin dans le champ de vision. Si la connotation du terme contribue à l'atmosphère pesante qui se dégage de l'ensemble, on peut également interpréter la métaphore 
comme une projection corporelle du narrateur sur l'objet de la perception qui, quelle qu'en soit la taille, est toujours ramené à son échelle. Ainsi, les châteaux s'y font froncements de sourcils, les branches de candélabre, des langues («the tongues of a tall candelabrum »), alors que les volets se ferment comme des paupières («the heavy shutters of the room »/ «my lids remained thus shut »). Le fait que certaines de ces métaphores soient figées ( «the head of the bed») n'ôte rien au phénomène, venant renforcer l'idée qu'il s'agit là d'un processus courant d'appréhension du sens qui n'affleure que sporadiquement.

Quelques lignes plus bas, transparaît la forte réciprocité entre capacité visuelle et/ou intellectuelle et volonté, dans la coprésence des deux auxiliaires de modalité could et would: « that I now saw aright I could not and would not doubt » (Poe 1842, 203). A la fin du texte, le phénomène apparaît de façon plus manifeste encore puisque c'est le refus de voir qui empêche le peintre de prendre conscience du danger auquel il expose sa bien-aimée: «And he would not see that the tints which he spread upon the canvas were drawn from the cheeks of her who sat beside him » (190). Le caractère impérieux de la pulsion artistique du peintre obnubilé par son œuvre, est souligné par la forme en italiques. Le mouvement ascendant, de l'objet de la perception au sujet pensant, est toujours concomitant d'un mouvement descendant par lequel c'est le sujet qui en définitive se projette dans ce qu'il perçoit pour y injecter un sens à son image: « [...] nous devons appréhender l'organisme et l'environnement dans la sélection et la détermination réciproques qui les lient l'un à l'autre » (Varela, Thompson \& Rosch 1993, 236). Cela nous mène au dernier moment de décodage du signe : le passage au signifié.

En dernier ressort, l'œil doit dépasser le signe pour accéder au signifié, apprentissage antérieur à la lecture qui se fait justement grâce aux livres d'images. Dans le texte de Poe, les trois moments précédemment décrits (projection dans le signe, déplacement dans l'espace phrastique et dépassement de la lettre) sont tous suggérés par le motif de la vignette, deux fois signalé dans le texte par des italiques: «done in what is technically termed a vignette manner », «the peculiarities of the design, of the vignetting, and of the frame » (189). Or, selon une de ses acceptions, « a small ornamental design filling a space in a book or carving, typically based on foliage » (Oxford English Dictionary 1999, 1598), le terme renvoie à une illustration en miniature à l'orée du texte. On pense ici aux lettres ornées ou aux enluminures destinées à embellir les manuscrits médiévaux :

Le traitement plastique de la lettre est souvent figuratif, selon des modalités variables dans le rapport avec le texte: dans la lettre «figurée », l'image incluse (par exemple un visage) n'a pas de rapport précis avec le texte [...] la lettre «historiée » réalisa l'association le plus intime de l'écrit et de l'image en intégrant celle-ci dans l'écriture même du texte. (Bergez 2004, 122 ; citation d'Hélène Toubert. Mise en page et mise en texte du livre manuscrit.) 
Dans ces manuscrits, les lettres situées en tête de chapitre ou de paragraphe pouvaient donc contenir un visage. Comment ne pas songer à la forme ovoïde du $O$ dans «The $\boldsymbol{O}$ val portrait»? l'ovale d'un cadre censé contenir un visage justement. Là, le signe linguistique concentre iconicité et arbitraire comme pour mieux opérer la transition vers l'abstraction. Le $O$ est une ouverture, un interstice, un judas, un œil conviant le lecteur à s'immiscer dans le texte. Le motif de la vignette l'enjoint à se faire tout petit pour s'enfoncer dans l'espace phrastique par l'image, à se faire «pupille » (étymologiquement une petite poupée, un être miniature), tout entier contenu dans l'œil qui cible, se focalise pour mieux accéder au monde que désigne le texte. L'œil déchiffre et au fur et à mesure que progresse la lecture, ne voit plus l'objet concret, la lettre, mais un tout diffus mêlé d'impressions éparses qu'il faut sans cesse réagencer autour d'un centre ponctuellement fixe alors que la périphérie s'estompe. Ce qui nous renvoie à une autre définition du mot vignette en anglais : "a small illustration or portrait photograph which fades into its background without a definite border » (Oxford English Dictionary 1999, 1598). Celle-ci apparaît comme une image sans cadre, une image perçue comme un texte.

\section{La théorie du double codage}

Le lien mots/images endogènes/images exogènes a été exploré de façon très approfondie dans le domaine de la psycho-cognition, notamment par Allan Paivio qui a élaboré la théorie du double codage. À partir de phénomènes mnémotechniques où des images très concrètes permettent la mémorisation de notions plus abstraites de type linguistique, ce dernier établit une distinction entre logogens et imagens. Tandis que le premier terme renvoie à des représentations mentales d'origine linguistique et multimodales, c'est-à-dire émanant de processus tant auditifs (la perception d'énoncés), visuels (la lecture) que sensori-moteurs (l'articulation orale, l'écriture), le second désigne des représentations mentales d'origine non verbale (perception d'un objet, ou d'images par exemple), elles aussi multimodales. Paivio montre que logogens et imagens empruntent des voies neuronales distinctes mais connexes, combinant des sous-structures autonomes (logogens visuels, auditifs, haptiques etc.) puisqu'une personne souffrant d'alexie (altération de l'aptitude à la lecture) restera capable de reconnaître des unités sémantiques auditivement, alors que celles atteintes d'anomie reconnaissent les objets mais sont incapables de les nommer, etc. Cette indépendance relative entre logogens et imagens et entre les sous-systèmes qui les composent n'interdit cependant pas que les deux œuvrent de conserve puisque c'est justement leur action séparée mais combinée qui 
facilite la mémorisation des mots concrets grâce à des processus cumulatifs. Ainsi les mots concrets seront plus aisément mémorisés que les notions abstraites car ils génèrent à la fois des logogens et des imagens :

[...] nonverbal and verbal codes, being functionally independent, can have additive effects on recall. For example, participants in free recall experiments are likely to name presented objects covertly and thus create a nonverbal (pictorial) and a verbal memory trace. They can also set up a dual verbal-nonverbal memory trace by imaging to concrete words, but this is somewhat less likely than naming pictures, hence the lower memory for concrete words than pictures. Abstract words are difficult to image and hence are least likely to be dually coded. The expected additive memory benefit of dual coding has been confirmed in numerous experiments (e. g., Paivio, 1975; Paivio \& Lambert, 1981), which also suggested that the nonverbal code is mnemonically stronger (contributes more to the additive effect) than the verbal code. (Paivio 2006, 4)

La théorie du double codage met au jour toute la complexité des phénomènes en jeu dans notre appréhension du réel par l'images, la parole et l'écriture. Surtout elle met l'accent sur le caractère multimodal de ces entités verbales et non verbales qui engagent des processus auditifs, visuels et sensorimoteurs dont on garde une trace au moment où ces représentations sont réactivées. Chaque fois, un complexe entrelacs sensoriel se met en place au moment de la perception d'un son, d'une image ou d'un graphème.

Afin d'illustrer ceci, il convient de revenir sur le passage où le narrateur requiert de son valet qu'il ferme les volets afin de plonger la pièce dans la pénombre pour s'isoler du monde extérieur, mieux contempler les tableaux qui s'y trouvent et parcourir le livret posé comme par un fait exprès sur un coussin à proximité. Que se passe-t-il à ce moment précis ? Les conventions d'écriture qui régissent le texte fictionnel exigent du lecteur qu'il adhère spontanément à ce qu'on lui propose, selon la célèbre expression de Coleridge («the willing suspension of disbelief »[1817, 145]). Ici le narrateur plante un décor fantasmagorique dont il nous faut, le temps de la lecture, considérer qu'il existe ou a existé (le texte est au prétérit). Les yeux grand ouverts, fixés sur le texte, nous voici alors nous aussi plongés dans une pénombre intérieure imputable en grande partie au mot night avec tout ce qu'il connote : impression de cécité, torpeur, voile enveloppant, anxiété, sens affûtés, membres engourdis, etc. Insensiblement, nous nous prenons à distinguer la clarté vacillante de quelque bougie. Si tout ceci se veut immatériel et fugace, il se produit néanmoins un phénomène qui n'a rien d'irréel. L'écrivain, qui est aussi son propre lecteur, trace des lettres sur le papier, relie les termes, tisse une trame dont la réalité est indiscutable et les effets tout à fait palpables, puisque le lecteur, en parcourant la page imprimée, rejoue mentalement chaque étape de cette construction (y compris parfois graphiquement). Simplement ce ressenti relève de réactivations multimodales médiées à partir de formes verbales auxquelles la vision s'est d'abord brièvement heurtée comme à ces petites flammes dont l'éclat inhibe 
ponctuellement la visibilité alentour. Le perçu immédiat est verbal, mais le perçu médié est constitué d'images endogènes qui condensent pour ainsi dire un vécu mémoriel privé, ressurgi de notre passé :

\begin{abstract}
The perceptual recognition and imagery entail activation of different sets of neural representations. In perception, an object directly activates an internal representation from a relatively small set by something like a template-matching process whereas in imagery the object name indirectly activates an imagen from an indefinitely-large set of imagens of the same class. The final outcome is determined by contextual cues and recent or remote past experiences with similar objects. (Paivio 2007, 144-145)
\end{abstract}

Le lecteur poursuit sa lecture et cette progression se fait alors même que germent en lui des représentations mentales qui se déploient puis se dissipent telles des volutes de fumée. Ainsi le contexte fictionnel auquel renvoient les signes détermine les conditions dans lesquelles s'opère le traitement concret du texte, fondé sur une oscillation constante entre perception du signe et élaboration du signifié. La lueur frémissante des flammes se confond alors avec les sons et les lettres qui dansent sur le papier: «I placed it so as to throw its rays more fully upon the book » (188). L'inquiétante métaphore des branches du candélabre désignées par le mot «tongues » revêt ici un sens nouveau où élocution (psychomotricité) et incandescence (phénomène visuel et tactile associant chaleur et luminosité) se relaient. Cette vision animiste suggère de nouveau que ce que je perçois du monde n'est qu'une extension de moi-même ; en retour on a ici l'impression que c'est le candélabre qui vient lécher l'œil du lecteur comme pour lui brûler la pupille (l'effet «dompte-regard» du portrait semble exercer la même action douloureuse : «I glanced at the painting hurriedly, and then closed my eyes » (189). La lecture ordinaire génère toujours ces synesthésies fécondes du fait de la multimodalité même des logogens et des imagens. Cependant, dans le cas d'un texte littéraire où l'écrivain façonne son récit de manière à lui conférer l'expressivité la plus juste, les effets de style rejouent pour ainsi dire à la surface du papier, ce qui se produit par ailleurs de façon souterraine. La formulation en épizeuxes que scande le narrateur est à ce titre éloquente : «Long, long I read - devoutly, devoutly I gazed» (188). Le phénomène d'écholalie reproduit textuellement la résonance articulatoire qui accompagne toute perception verbale. D'une certaine manière, ces figures indiquent aussi stylistiquement que, du fait même de leur cinétisme, les signes se nourrissent de ceux qui les ont précédés. La structure en partie homothétique de l'enchaînement des signes qui s'appellent les uns les autres transparaît concrètement dans la duplication du signe. De fait, le premier terme de la répétition renvoie à un signifié conventionnel relativement fixe, mais comporte aussi un deuxième signifié, co-textuel celui-là : le signe qui le suit et qui se substitue à lui du fait du cinétisme qui anime l'ensemble (cf. le concept d'indexicalité). De surcroît, comme ici le deuxième terme est identique au 
premier, la présentation graphique est littéralement re-présentée. Signe et signifié sont dans un rapport d'isomorphisme absolu, à ceci près qu'ils ne sont pas perçus simultanément et sont donc focalisés successivement; d'où la métaphore de l'homothétie car elle correspond à une projection géométrique où la même forme se répète en subissant un agrandissement ou un rétrécissement. Au-delà, la structure en épizeuxe, deux fois répétée, montre la capacité de la langue à s'autoportraiturer aussi sûrement que s'il se fût agi d'un reflet pictural. Or précisément, dans ce segment, nous avons affaire à une mise en miroir lecture du texte/contemplation du tableau : «Long, long I read - and devoutly, devoutly I gazed. » Là, le tiret, charnière typographique, visuelle donc, sépare et relie tout à la fois. Il est relayé par la conjonction de coordination and, élément linguistique à valeur exclusive ou inclusive. La ligature ou la séparation sont donc à la fois visuelles et conceptuelles. Poe glisse une micro-image, un fragment de cadre, dans le texte par le truchement d'un signe de ponctuation en apparence anodin.

Le phénomène de mise en abyme qui caractérise le conte - le texte aborde le thème de la lecture alors même qu'il est objet de lecture, révèle la nature multiple du signifié textuel à la fois conventionnel et stable, contingent et dynamique ; une sorte de feuilleté de sens, "manifold and multiform » (Poe $1842,203)$. Ainsi, quand le narrateur demande à son valet de clore les volets et d'ouvrir les tentures du lit à baldaquin, il souhaite créer une atmosphère propice à la contemplation des tableaux qui ornent les murs de la pièce dans laquelle il se trouve. Mais il décrit (ou plutôt $s^{\prime} y$ dit en filigrane) le protocole qui préside à la lecture du texte qui le contient : obturation sensorielle d'une part - il faut se fermer à tout élément susceptible de venir détourner l'attention du texte - puis lever de rideau sur un spectacle désormais intérieur. C'est à cette condition que pourra opérer la magie, c'est-à-dire l'effacement du signe derrière le signifié.

\section{Conclusion}

Sous couvert de décrire l'effroi suscité à la vue d'une œuvre picturale symbole de fixité et d'instantanéité perceptuelle ainsi que d'identité de conformation, Poe instaure un dialogue texte/image par le seul biais de l'écriture. Pour ce faire, il y joue à la fois sur le dynamisme propre au signe linguistique, sur les effets sonores que ce dernier génère par iconicité, et sur le style qui permet de redoubler tout en les illustrant, des phénomènes de transfert de sens par ailleurs toujours présents linguistiquement. L'on peut de fait envisager la nouvelle comme l'évocation littéraire d'un transfert de sens du perçu au représenté puis de l'image au texte et retour. D'ailleurs, le thème du 
vampirisme auquel renvoie l'ultime antithèse Life/dead ${ }^{3}$ s'inscrit au cœur du texte comme métaphore des phénomènes intersubjectifs qui s'y jouent (transfert de vie du modèle à la copie, du sujet à l'objet, transfert de sens du narrateur au lecteur, etc.), mais aussi du passage du visuel, ou plus généralement du perçu, au concept et au lu, lui-même permettant un accès à l'imaginaire du poète et du lecteur.

Ces quelques réflexions vont, dans un prochain article, nous amener à résoudre deux énigmes souvent relevées à propos de cette même nouvelle : d'une part, celle de la raison d'être du valet (valet qui disparaît aussi bizarrement qu'il était apparu) et, d'autre part, celle de la blessure du narrateur, autant d'éléments de l'intrigue que Monique Dubanton qualifie d' «anomalies » $(1979,104)$. Nous verrons que tous deux contribuent en réalité à accélérer le passage au signifié, à rendre pour ainsi dire le signe plus «transparent » grâce à l'acte d'animation nécessaire à la construction du sens (Belting 2004, 43-44).

3 Rappelons ici que le titre initial de la nouvelle était « Life in death » [...] » (Lévy 2004, 11). 


\section{Bibliographie}

\section{Corpus littéraire}

PoE, Edgar Allan, (1842) 1990. Tales of Mystery and Imagination, London, Everyman.

\section{Autres ouvrages cités}

Belting, Hans, 2004. Pour une anthropologie de l'image. Paris, Gallimard.

Bergez, Daniel, 2004. Littérature et peinture. Paris, Armand Colin.

COLERIDGE, Samuel Taylor, (1817) 1905. Biographia literaria, or, Biographical sketches of my literary life and opinions. London, George Bell \& sons.

DeHAENE, Stanislas, 2007. Les neurones de la lecture. Paris : Odile Jacob.

Dubanton, Monique, 1979 (102-110). «L'Ovale du portrait. La fonction de l'écriture chez Edgar Poe ». Poétique, $\mathrm{n}^{\circ} 37$.

Hochberg, Julian \& Brooks, Virginia, 2007 (139-147). «Reading as an intentional behavior ». In the mind's eye: Julian Hochberg on the perception of pictures, films, and the world. Oxford, OUP.

LECERCLE, Ann, 1990 (77-91). «L'Inscription du regard. » Du fantastique en littérature : figures et figurations. Aix-Marseille : Presses Universitaires de Provence.

LEHRER, Jonah, 2008. Proust was a Neuroscientist. Boston, Houghton Mifflin.

LETALLEUR-SOMMER, Séverine, 2009 (267-281). «Dialogues théoriques autour de l'émergence du sens linguistique et visuel ». Anglophonia Sigma ${ }^{\circ} 26$, Toulouse, Presses Universitaires du Mirail.

LEVY, Paule, 2004 (9-22). «La question de la clôture narrative dans The Oval portrait d'Edgar Allan Poe ». Journal of the Short Story in English $\mathrm{n}^{\circ} 42$, Angers, Presses Universitaires d'Angers. Oxford English Dictionary. Oxford: OUP, 1999.

PAIVIO, Allan, 2007. Mind and its Evolution. A Dual Coding Theoretical Approach. Mahwah, NJ, Erlbaum.

2006. "Dual coding theory and education." Draft chapter for the conference on "Pathways to Literacy Achievement for High Poverty Children," The University of Michigan School of Education.

http://www.umich.edu/ rdytolrn/pathwaysconference/presentations/paivio.pdf 
SAdOSKI, M. \& PAIVIO, A., 2004 (1329-1362). "A dual coding theoretical model of reading.” In R. B. Ruddell \& N. J. Unrau (Eds.), Theoretical models and processes of reading. Newark, DE, International Reading Association.

VAN DE WIELE, L., 2005. «Le rôle cognitif du dessin dans les manuscrits poétiques de Jean Cocteau ». Degrés. M. Dominicy et D. Gullentops (dir.), Bruxelles, $\mathrm{n}^{\circ}$ 121-122, cité dans Callebaut Alex «Jean Cocteau et la ligne transgressée » Textimage, Revue d'étude du dialogue texte image, $\mathrm{n}^{\circ} 4$, L'image dans le récit.

http://www.revue-textimage.com/sommaire.htm (lien consulté le 23 juin 2012). 